\title{
Die Inzidenz der Demenz ist rückläufig
}

\author{
Angesichts der demografischen Entwicklung werden düstere Szenarien über die Häufigkeit \\ der Demenz in naher Zukunft an die Wand gemalt. Studien sprechen eine andere Sprache: \\ Die Schlüssel zur Lösung des Problems dürften bereits bekannt sein.
}

In der Folge der zunehmenden Lebenserwartung wird auch ein gewaltiger Anstieg der Demenz erwartet. Untersuchungen aus der jüngsten Zeit weisen aber darauf hin, dass die altersspezifische Inzidenz der Demenz in den hoch entwickelten Ländern zurückgeht. Derartige zeitliche Trends können erfahrungsgemäß am besten durch ein fortlaufendes Monitoring der Bevölkerung über lange Zeiträume hinweg unter Verwendung etablierter diagnostischer Kriterien erfasst werden.

Genau diese Voraussetzungen sind in der seit 1948 laufenden Framingham Heart Study gegeben. Seit 1975 wurde in das Untersuchungsprogramm auch eine Gruppe von 5.205 damals über 60 Jahre alte Personen aufgenommen. Diese Nachkommen der ursprünglichen Kohorte haben mittlerweile neun Untersuchungstermine im Abstand von vier Jahren hinter sich. Seit 1981 gehört auch eine Mini Mental State Examination
(MMSE) zum Programm. Das altersund geschlechtsadjustierte kumulative Fünfjahresrisiko für eine Demenz betrug - jeweils pro 100 Personen - in den Untersuchungsperioden:

_ späte 70er-, frühe $80 \mathrm{er}-J a h r e: 3,6$

_ späte $80 \mathrm{er}$-, frühe $90 \mathrm{er}$-Jahre: 2,8

_ späte 90er-, frühe Nullerjahre: 2,2

_ späte Nuller-, frühe 10er-Jahre: 2,0.

Im Vergleich zur Inzidenz während der ersten Untersuchungsperiode ging die Inzidenz in den späteren Perioden um $22 \%, 38 \%$ und $44 \%$ zurück. Diese Risikoverminderung wurde aber nur bei Personen beobachtet, die es in ihrer Bildungslaufbahn mindestens zu einem höheren Schulabschluss gebracht hatten. Ihr Risiko für eine Demenz war im Vergleich zu Personen mit niedrigerem Bildungsgrad um 23\% geringer.

Mit Ausnahme von Adipositas und Diabetes gingen im Verlauf der Studie auch kardiovaskuläre Risikofaktoren für eine Demenz zurück, etwa Schlaganfall,
Vorhofflimmern oder Herzinsuffizienz. Allerdings war keiner dieser Trends ausreichend ausgeprägt, um den Rückgang der Demenz-Inzidenz zu erklären.

- Satizabal CL, Beiser AS, Chouraki Vet al. Incidence of dementia over three decades in the Framingham heart study. N Engl J Med. 2016;374:523-532

\section{KOMMENTAR}

Die Ergebnisse der Untersuchung berechtigen zu einer gewissen Hoffnung, dass es mit der Demenz nicht so schlimm kommen wird wie befürchtet, da es offensichtlich Mittel zur Prävention gibt. Körperliche und geistige Aktivität scheinen sicher nicht zu schaden, vielleicht sogar erheblich zu nützen. Gleichzeitig zeigt die Untersuchung, wie wenig wir über die komplexen Einflüsse unserer Lebensweise auf die körperliche und geistige Gesundheit wissen. Selbst retrospektiv können wir nur spekulieren - von sicheren prospektiven Aussagen sind wir auf Populationsbasis weit entfernt.

Prof. Dr. med. H. S. FüeßI

\section{Ist das überhaupt noch derselbe Mann?}

\begin{abstract}
Wegen allgemeiner Schwäche wurde ein 76-jähriger Mann ins Krankenhaus aufgenommen. Einige Wochen zuvor war ihm und seiner Umgebung eine zunehmende graublaue Verfärbung der gesamten Haut aufgefallen. Diese war so ausgeprägt, dass der zuvor weißhäutige Mann nicht mehr anhand seines Personalausweises zu erkennen war. 14 Monate zuvor war ein malignes Melanom diagnostiziert worden, das BRAF-negativ war. Fünf Monate vor der Vorstellung wurden multiple Metastasen in Leber und Milz festgestellt. Der Mann wurde mit vier Zyklen Dacarbazin behandelt. Die ausgeprägte Hyperpigmentierung der Haut war Ausdruck einer diffusen Melanosis cutis. Diese Veränderung ist ein prognostisch ungünstiges Zeichen und geht mit einer durchschnittlichen Überlebenszeit von etwa sechs Monaten einher. Der Patient lebte aber nach Auftreten der Melanosis cutis noch 16 Monate, möglicherweise als Ergebnis einer Immuntherapie mit Ipilimumab.
\end{abstract}

Prof. Dr. med. H. S. FüeßI

- Jansen T, HoffNP (norman-philipp.hoff@med.uni-duesseldorf.de).Diffuse melanosis cutis. NEnglJ Med. 2016;374:1177

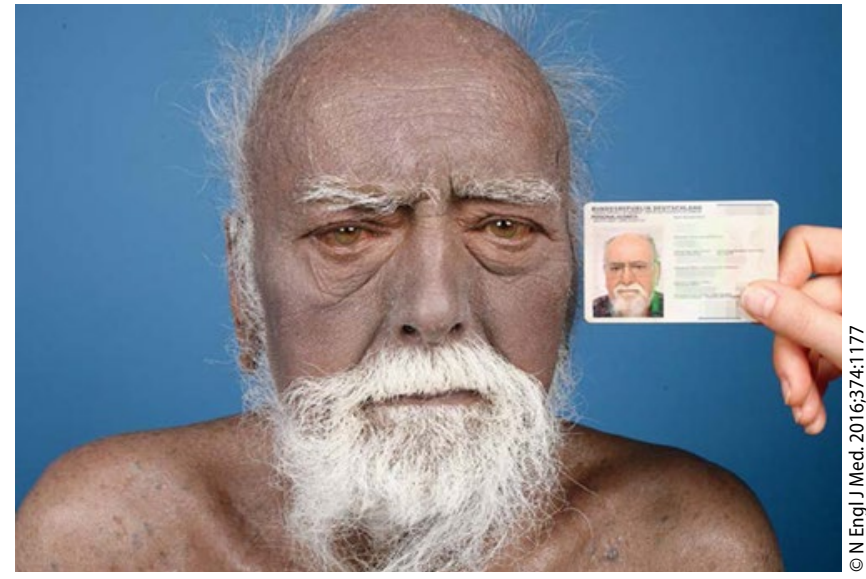

Ausgeprägte Hyperpigmentierung der gesamten Haut; Personalausweis zum Vergleich. 